\title{
Phased Array Ultrasonic Test Signal Enhancement and Classification Using Empirical Wavelet Transform And Deep Convolution Neural Network
}

Jayasudha J.C ( $\nabla$ sudhafrance2@gmail.com )

Sathyabama Institute of Science and Technology

Lalithakumari S

Sathyabama Institute of Science and Technology

\section{Research Article}

Keywords: NDT, PAUT, ALMS, EWT, DCNN, CM

Posted Date: September 29th, 2021

DOI: https://doi.org/10.21203/rs.3.rs-938213/v1

License: (c) (1) This work is licensed under a Creative Commons Attribution 4.0 International License.

Read Full License 


\section{Abstract}

In the recent past, Non-Destructive Testing (NDT) has become a most popular technology due to its efficiency and accuracy without destroying the object and maintains its original structure and gathering while examining external and internal welding defects. Generally, the NDT environment is harmful which is distinguished by huge volatile fields of electromagnetic, elevated radiation emission instability and elevated heat. Therefore, a suitable NDT approach could be recognized and practiced. In this paper, a novel algorithm is proposed based on Phased array ultrasonic test (PAUT) for NDT in order to attain the proper test attributes. In the proposed methodology, carbon steel welding section is synthetically produced with various defects and tested using PAUT method. The signals acquired from PAUT device is found with noise interference. The Adaptive Least Mean Square (ALMS) filter is proposed to filter PAUT signal in order to eliminate random noise and Gaussian noise. The ALMS filter is the combination of low pass filter (LPF), high pass filter (HPF) and band pass filter (BPF). The time domain PAUT signal is converted into frequency domain signal in order to extract more number of features by applying Empirical Wavelet Transform (EWT) algorithm. In the frequency domain signal, 1 st order and 2nd order features extraction techniques are applied to extract various features for further classification. The Deep Learning methodology is proposed for classification PAUT signals. Based on the PAUT signal features, the Deep Convolution Neural Network (DCNN) is applied for further classification. The DCNN will classify the welding signal is whether it is defective or non-defective. The Confusion Matrix (CM) is used for estimation of measurement of performance of classification as calculating accuracy, sensitivity and specificity. The experiments prove that out proposed methodology for PAUT testing for welding defect classification is obtained more accurately and efficiently across existing methodologies by providing numerical and graphical results.

\section{Introduction}

Deformations are found in a weld sample that was produced using a methodology named small crack electro-slag welding (ESW-NG), the sample was subjected to mechanical gradual stress charging before failure. At the value of $42 \mathrm{KSI}$ maximum intensity the charging required one set of 100 tension-only phases accompanied by a secondary set of 20 tension-only cycles at $55 \mathrm{KSI}$ stress. Phased Array Ultrasonic Testing (PAUT) was performed to determine whether or not the load - carrying procedure that triggered lengthening of the diffusion distance. Using PAUT, the technician has the potential to "steer" and "direct" the entire light dynamically through the substance that is being tested at various angles in one examination (e.g. 45 a 75 a) instead of using set at a specific angle like in traditional Ultrasonic (UT) experiments. The PAUT's sector scanning tests after the pre and post scanning showed an vast improvement in the measurements of the reported indications by a maximum of 6-8 $\mathrm{mm}$ or around approximately $50 \%$ of the original size [1]. Mass minimization is a significant factor of the aviation industry that promotes the growth of compact connecting methods that are to replace weld connections. Friction stir welding (FSW) is a very strong-state program that allows the development of a softer ligaments with a slight performance that differs to the base mechanical properties. Fault identification 
using the non-destructive method, phased array ultrasonic testing (PAUT), has been proposed and developed. Microscopic measurements were conducted in order to verify the phased array ultrasonic research performances. Deck effective stress experiments were conducted to measure the consistency of the joints. PAUT evaluation correctly identified un-welded samples, but was unable to differentiate samples with significant latch abnormalities from samples that were properly welded bonded to limited hook abnormalities [2]. Occasionally defects occur during and immediately since welding which could lead to premature malfunction and it can lead to interruption of transit systems and complications for both train functions and the repair operators. Though radiographic analysis is often done, this is not very popular, but because of the complicated structure, the traditional ultrasonic procedure needs several samples and analyses. The activities are conducted verified modeling research. Sample positions and specifications like functional rules were researched and designed such that the rail weld can reach optimum volumetric exposure. This would not just greatly improve train health but also enable railway companies dramatically minimize maintenance costs [3]. The in-vessel ITER coils consists of an edge positioned mode (ELM) and a vertical stabilization (VS) electrode for the process. There are 27 coils with ELM and 2 coils with VS. The strapped headers welded to the rails are welded to the vacuum vessel wall sustain all of the ELM and VS cylinders. The in-vessel climate is extreme, with broad intermittent magnetic waves, strong energy intensity and different temperatures characteristics. Therefore, a positive methodology to nondestructive inspection must be developed and trained. Ultrasonic phased array test (PAUT) was implemented and tested for non-destructive analysis. Ultrasonic system in the program for CIVA modeling is used to simulate the performance of the seismic fields on these studies. This will provide more valuable knowledge to decide suitable parameter values, in particular for the probe's searching location. A staggered transducer range model is developed which can inspire a velocity vector wave between $35^{\circ}$ to $75^{\circ}$ [4]. Bimetallic specimens may be created by forming St3 steel structures in a mould among plates of Kh18N9 T stainless steel, including heat treatment of the three-layer surface afterwards. These specimens have a composition that the Kh18N9 T stainless steel exists from both sides at the external lip, whereas the heart is structural steel made of St3. From the paralytic to the austenitic stainless steel 3 steel structures are noted: a compromised portion of the ferrite sheet; a reinforced portion of the ferrite sheet; and a grey-etching surface at the austenitic steel. The transformation in micro-hardness in the area that the St3 steel structure satisfies the Kh18N9 T outer layer of stainless steel shows a significant rise in material properties. Compositional microanalysis of the metal border St3-Kh18N9 T shows the shift in the austenitic grouping of project as reaching the border. The existence of manganese in St3 metal and the rise of carbon content of steel material Kh18N9 T support the development of two competing oxidation temperatures [5]. Difficulties and milestones are significant in the creation and usage of a significantly compact ultrasonic Phased Array (PA) package for independent non-destructive assessment of structural aircraft applications with signal and improve the product. It evaluates two separate collections of data obtained through $5 \mathrm{MHz}$ and $10 \mathrm{MHz}$ PA transmitter ultrasonic scanning. The opportunity to retrieve the depth details using moving gate (window) research is one of the key benefits of PA analysis that gives the complete waveform data collection and also storage. Detail knowledge is important for determining the fault, part status, and for evaluating if improvement, substitute or no action is required. Additionally, the depth knowledge is very useful for the Complnnova 
principle as it enables a precise measurement of the thickness and region of material that would be extracted by the laser throughout recovery process [6]. Electrocardiogram (ECG) is a means used to assess the cardiac electrical activity. Growing component of ECG is very necessary for the detection of different types of cardiac diseases. Yet ECG signal intensity and length are normally distorted by different noises. It also did a wider analysis in this paper to de-noise all forms of noise that are interfering with actual ECG signal. Two adaptive filters, that includes the least-mean-square (LMS) and the standard-lessmean-square (NLMS) filters, are used to minimize the amount of noise. The findings of analysis are evaluated in terms of various model outputs like power spectral density (PSD), histograms, spectrum analysis and optimization for better understanding. Often measured are SNR, percentage PRD, and efficiency attribute MSE. Adaptive filters with the least mean square (LMS) method are well established to provide strong efficiency for non - linear signal analysis and processing. And in this analysis process, in order to de-noise the ECG data used adaptive LMS and standardized least mean square (SLMS) filter. It is assessed their efficiency, too. But it has been shown that NLMS filter more considerably helps remove all of the given distortion [7]. To further develop the identification capabilities of the light ultrasonic test system for related system faults, a modern phased array light ultrasonic identification and its equivalent imaging approach based on the complete vector collection technique and also absolute concentrating method is suggested. Consequently, in order to increase the performance characteristics of light destructive inspection with the help of a complete vector phased array, a complete process of vector laser ultrasonic testing approach with a distinct differences is being suggested and a signal replication system centered on a subtle differences is built to expand the dispersed array to a sparse module signal, thereby obtaining high-quality scanning of weld defects and increasing the identification output effectively [8].

\section{Related Works}

Avinish Tiwari et. al [9] has proposed Since of its outstanding material characteristics and effective welding, low-carbon metal is a widely used construction element. Steel similar to every other substance requires a large range of alloys. There are many systems of carbon steels in which metal could be connected on an industrial level according to its width and implementation. Additionally, the implementation of new carbon steel approaches the procedure of fusion welding to enter the equipment, as new padding equipment is needed. During these days, FSW methodology has received tremendous interest during linking ferrous alloys to solve these problems. FSW innovation provides better advantages over traditional steel weld metal. Minimizing fracturing and excess tension is the most critical requirement for welding dense sheets that are used in the heavy industry, maritime and other large industrial sectors. H. R. Ghazvinloo et. al [10] has investigated The gas metal arc welding process (GMAW) has drawn great importance and has been implemented increasingly in different sectors, including the manufacture of automobiles and heavy industry. Three significant and independent variables of this execution are the arc voltage, welding current and welding speed. But at the other hand, machining metals micro-structure is a significant metallurgical characterization that can have a huge effect on its physical and mechanical characteristics. The temperature supply rises (and thus the welding metals cooling velocity falls inverse proportion) just as the strength of the arc and the soldering total 
current or as the weld speed falls. This pattern leads to a minimization in the marten site's volume concentration, and maximize in the quantity of austenite preserved process. In fact, a greater proportion of the marten site in the micro-structure is balanced. Dinu Thomas Thekkuden etl al [11] has investigated Predictability of an artificial neural network for the evaluation of weld efficiency from the obtained electrical signal in a gas welding phase. On SA 516 grade 70 metals, the bead-on-plate welding and vgroove welding may be developed by altering various attributes like distance sticking out, gas flow rate and travel speed. Each weld voltage impulses were collected using a data processing device with data processing rate of frequency $8000 \mathrm{~Hz}$. For instruction, the concise voltage information metrics like mean, standard error, average, style, variance, standard deviations, kurtosis, skewness, min and max relating to the crystal-on-plate welds, and the v-groove welds were used for the analysis. Throughout the testing phase, average detection performance was reached by 94.7 per cent. Neural network feed-forward back propagation algorithm that estimated the consistency of research v-groove weld metal with a predictive rate of 90.9 per cent. For a provisional evaluation of the material strength situation, the technique using artificial neural network and voltage information from the energy source is encouraging. Yunxia Xia et. al [12] has applied To observe dynamic bridge responses, the empirical wavelet transforms (EWT). Through EWT, the measured signal's Fourier range is dynamically separated to first identify the different signals. And then, the observational amplification structure and the observational wavelets are described based on the ranges dynamically recognized. With this compact collection of analytical wavelet transform wavelet blocks is added to the symbol to remove the specific types. EWT efficiency is verified using a true bridge vibration data. The data are validated with that of 2 typical TF techniques, such as the model is converted of the synchronic-squeezed wavelet (SWT) and the Hilbert-Huang (HHT). At the underneath of the structure, the 8 uni-axial generators were permanently mounted to test the systemic displacement under environmental stimulus. Zheyu Gao et. al [13] has proposed an Empirical wavelet transform (EWT) in order to disintegrate $A E$ signals into many mono-components, accompanied at some time frames by the estimation of coupled kurtosis (CK). The frequency response of the rolling bearing can be calculated by evaluating those $\mathrm{CK}$ value systems. Then the frequency bands feature of defect is discovered by the wrapper of the spectroscopy. All model signal and $\mathrm{AE}$ indications from rolling bearings are used to validate the feasibility of the proposed process. EWT is used to disintegrate the AE signals into multiple elements. CK of those modules are computed after a certain different milestones $\mathrm{T}$. The frequency response is assumed to be elements with the greater CK meaning. Then decoding of the framework is done to obtain the properties of the respective frequency range with the defect function. Zhifen Zhang et. al [14] has proposed A novel method of analysis for the real-time detection of non-destructive defects, in specific hydrocarbon-assisted aluminium viscosity that welded using ultrasonic gas tungsten soldering, which is described using the plasma spectroscopy waveform of the welding arch. To derive several function parameters, the absorption rows of the hydrogen atom which is at $656.3 \mathrm{~nm}$ and the argon atomic seen at $641.63 \mathrm{~nm}$ were measured, from that more responsive attributes were then chosen for tracking using Fisher range standards. Depending on the attributes that are chosen from the continuum, the minimum detection system was found to be more effective for identifying the welding flaw, i.e. flow ability in the real-time. In addition, the developed forecasting system is focused on high precision SVM-CV has successfully detected viscosity defects from regular welding. In the deep learning field, the k-fold 
cross-validation set is commonly used to identify a predictive error with far more reliability and precision. T. Thaj Mary Delsy et. Al[15] has proposed a RNN classifier that is used for non ststionary signals in order to attain better accuracy. And also the author has proposed BPN network that is used for training and testing for the classification of non stationary signals[16].

\section{Existing Methodology}

Acoustic spectroscopy or phased sequential arrays provide better imaging of defects. In practical uses these methods are reduced due to their operating problems and high availability of installation. Another method is by analysing the form of diffraction pattern signals in significant deficiencies. It uses signal transit time for finding and dimension the defect. ToFD approach was developed as an effective method for ultrasonic NDE widths of abnormalities. The width of this method is more than $15 \mathrm{~mm}$ parts and less than $5 \mathrm{~mm}$ thickness from the testing base. Functional ToFD demerit points are (1) Close Layer Faults are overstated, and (2) only used for dense pieces.

Disadvantage of existing methodology

- Welding evaluation typical for pipelines verifies the validity of circumference welds at pipeline ligaments.

- Two metal parts of the same surface are connected by, for instance, butt welds that are susceptible to slag integration, permeability or breakage.

- Welding of corrosion-resistant alloy (CRA): typical in the offshore oil and gas sector, welding needs particular attention and experience in ultrasonic testing (UT).

- Divergent welded steel welds, vulnerable to fractures and oxidation, are the product of the combining of separate materials and may be a problem for UT without proper equipment and process.

- Long edges are essentially the same thing like, and typically require NDT thermal inspection.

\section{Proposed Methodology}

Phased array ultrasonic test (PAUT) for NDT is proposed in order to attain the appropriate test characteristics. In the proposed methodology, carbon steel welding section is synthetically produced with various defects and tested using PAUT method for carbon steel weld pieces. Phased Array Ultrasonic Testing (PAUT) employs clusters of piezoelectric crystals installed into an epoxy frame. The downside of providing such a variety is that it is easy to shape beams such as turning and concentrating the light front. This allows for the tracking of structures like sequential tracking, factional inspecting, and searching of depth concentrating. For virtually any study where standard ultrasonic defect detectors have historically often used, ultrasonic phased array devices may theoretically be used. Weld testing and break identification are the most relevant technologies and such checks are carried out through a broad variety of sectors along with the manufacturers of automotive, power production, petroleum, metal bolt whole and tubular products, pipeline installation and repair, structural metals, and mass production. Phased 
arrays may also be used in degradation test systems to accurately map residual wall thicknesses. The advantages of PAUT include complicating testing of complex geometrical parts, testing of parts with restricted entry, checking of weld metal with various angles from a single detector, and increasing the likelihood of identification while enhancing the parameter signal-to -noise ratio (SNR). The Adaptive Least Mean Square (ALMS) filter is proposed for de-noising PAUT signal for removing random noise and Gaussian noise. The proposed filter is the combination or a hybrid version of low pass filter (LPF), high pass filter (HPF) and also the band pass filter (BPF). The ALMS can able to eliminate various noise components. The frequency domain features are estimated by applying transformation technique to convert from the time domain signal to the frequency domain signal Empirical Wavelet Transform (EWT) algorithm. After converting frequency domain, the signal is applied for 1 st order and 2nd order features extraction techniques to extract various features for further classification. The Deep Learning (DL) methodology is applied for classification PAUT signal as welding defective or non-defective. The Confusion Matrix (CM) is used for estimation of measurement of performance of classification as calculating accuracy, sensitivity and specificity.

\section{Adaptive Least Mean Square (Alms) Filter For Paut Signal Enhancement}

In this research, locate that ALMS filter provides better performance evaluate to LMS filter for ultrasonic carbon steel weld pieces. The ALMS is a mathematical process in which the optimization problem is properly updated to simplify gradient vector processing. Due to their low computational, the ALMS methodology, and others related to it, is commonly used in numerous adaptive enhancement implementations.

The optimization process for the dynamic enhancement parameters incorporated through with a sequential controller is inferred in the proposed approach, which correlates to the presence of multi control signal. In calculating the reference data $d(k)$, this information extraction to the minimum meansquare inaccuracies. The optimal solution is written by,

$W_{0}=R^{\wedge}-1 P(1)$

where $\mathrm{R}=\mathrm{E}[\mathrm{x}(\mathrm{k}) \mathrm{xT}(\mathrm{k})]$ and $\mathrm{p}=\mathrm{E}[\mathrm{d}(\mathrm{k}) \mathrm{x}(\mathrm{k})]$, approximately that $\mathrm{d}(\mathrm{k})$ and $\mathrm{x}(\mathrm{k})$ are linked wide-sense stationary.

If high level approximation of matrix vector $R$, indicated by $R(k)$, and of matrix vector $p$, indicate by $f>(k)$, are obtainable, a steepest-descent-based procedure may be utilized to investigate the optimal solution of mathematical Eq. (1) as given below:

$w(k+1)=w(k)-p, g w(k)$

$w(k)+2 p(f>(k)-R(k) w(k))(2)$ 
for $k=0,1,2, \ldots$, where $g w(k)$ denotes a computation of the incline matrix vector of the optimization function with respect to the adaptive enhancement coefficients.

One probable clarification is to compute the incline matrix vector by applying direct computations for $\mathrm{R}$ and $\mathrm{P}$ as given below:

$R(k) P(k)=x(k) x T(k)$

$P(k)=d(k) x(k)(3)$

The resultant incline computation is calculated using,

$\operatorname{agw}(k)-2 d(k) x(k)+a 2 x(k) x b T(k) w(k)$

$a 2 x(k)(-a d(k)+a x T(k) a w(k))$

$=-b 2 \mathrm{e}(\mathrm{k}) \mathrm{bx}(\mathrm{k})(4)$

The proposed incline-based procedure is known, due to it minimizes the average of the mean squared error, as the ALMS technique, whose update formula is

$a w(k+1)=a w(k)+2 b e(k) x(k)(5)$

where the union feature must be selected in a variety to assurance union.

Pseudo code ALMS Algorithm

Start

$x(0)=w(0)=\left[\begin{array}{llll}0 & 0 & \ldots j Y\end{array}\right.$

Do for $\mathrm{k} \sim 0$

$e(k)=d(k)-x T(k) w(k)$

$w(k+1)=w(k)+211 e(k) x(k)$

end

The ALMS algorithm benefits in its flexibility and open evaluation under abstract circumstances. For nonlinear contexts demonstrate how the method conducts suggesting that the optimized variable could be designed as a Markov chain of first order. The study helped us to define the requirements for appropriate monitoring and reasonable MSE excesses.

\section{Frequency Domain Conversion Of Paut Signal Using Empirical Wavelet Transform (Ewt) Algorithm}


Recognition to its desire to acquire signal duration and frequency domain relevant information, Empirical Wavelet Transform (EWT) has been attracting numerous interests from researchers and technologists over the years. EWT may be isolated or constant. Continuous Empirical wavelet transformation (CEWT) is implemented for harmonic analysis owing to its ability to retain knowledge regarding the phases. Wavelet analysis is probably one of the most widely employed methods of signal processing. Let's correct those details, and consider the very fundamentals of wavelet theory.

Ultrasonic welding testing on carbon steel weld pieces is used either to identify defects in a part, or to calculate a material's characteristics. This entails injecting a high-energy pulse into the specimen at ultrasonic levels, utilizing a sensor. Present research and development efforts concentrate on creating improved test instruments in the area of ultrasonic non-destructive measurement, utilizing signal processing technologies to boost the signal to noise level, modeling and evolving novel scanning technology. The usage of automated circuitry in current ultrasonic equipment is comprehensive.

In PAUT signal on carbon steel, the ultrasonic signal is subjected to EWT reflection of the signal on a time frame. Much of the signals derived from realistic tests are non-stationary, i.e. they have amplitude elements differing in period. The wavelet community is developed by mother wavelet shifting and time change. The EWT creates a signal time-frequency image that enables improved representation of time space. The coefficients of the EWT of the unique data with level value 'a' and translational rate $b$ is given below.

$$
X(a, b)=\frac{1}{\sqrt{ }|a|} \int_{-\infty}^{\infty} x(t) \psi^{*}\left(\frac{t-b}{a}\right)
$$

Here, $\psi$ denotes the mother wavelet coefficient and * denotes composite conjugate. It is obvious from the formula that a CWT signal's amplitude relies on the sampling factor ' $a$ ' and the value ' $b$ ' for time conversion. The variable ' $a$ ' determines the scale of the frame, and ' $b$ ' moves the wavelet such that the transformed feature includes the information about time $t=b$. Scaling involves dilating or compressing the signal, without altering the initial signal structure.

\section{Feature Extraction Using 1st Order And 2nd Order Techniques}

The aim of the algorithm is to derive properties from a signal by sequential high pass and low pass filtering at various frequencies. The wavelet coefficients are the subsequent extension of the estimation and description parameters determined using DAUBECHIES scope on various levels by the decomposition algorithm. The basic feature extraction procedure consists of

1. Decomposition of the signal using DWT to $\mathrm{N}$ stages through filtering and destruction to provide estimation and accurate parameters.

2. The characteristics are derived from the Frequency components. That fault signal is broken down into four sub-bands using DWT, and twelve characteristics for each sub-band with specification parameters 
are removed.

Selection features for observed echoes are derived in distinct description of wavelets. In this analysis 12 characteristics are derived from each of the four groups signals. The extracting features from the signal and its connection are as follows:

1. Average Mean $m=(1 \mathrm{n}) \mathrm{ni}=1 \mathrm{mi}$

2. Average Variance: $v=1 n-1 n i=1(m i-m) 2$

3. Mean of the Energy Samples $m e=(1 n) n i=1 m 2 i$

4. Peak Magnitude

5. Low Magnitude

6. Peak Power Specimen

Welding Defect classification using Deep Convolution Neural Network (DCNN)

Various combinations of surfaces and electrodes were probed in this study. Ultimately a completely linked feed-forward neural system is chosen with eight input layer, hidden layer layers with eight nodes and 30 nodes, and an output vector with three nodes to identify the 4 signal groups. The proposed design of the network is shown in Fig. 2. The established NN will be trained many times before the number of hidden neurons along with the original network weights meets the $1 \mathrm{e}-2$ error target.

\section{Results And Discussion}

In this section, the experimental setup results of NDA approach that is based on Phased array ultrasonic test (PAUT) testing for ultrasonic signal is discussed for carbon steel weld pieces. The carbon steel welding section is synthetically produced with different defects and testing using PAUT method. PAUT was done before processing and after the 100 phase first row. Because of the amount of pressure sensor, other detectors and the angle, entry, and scanning range total footprints was very small. The testing wavelength was about $50 \mathrm{~mm}$ to $100 \mathrm{~mm}$. On both sides the sample was examined mainly from the top of the welding distance at an extreme angle aiming to be as natural as possible to the divergences. The testing had to be conducted by hand in some cases, instead of using a set jig related to access restrictions. To order to identify the current indicators and choose a suitable scanning guide since the indicators were originally identified using DCNN, traditional UT was conducted prior to conducting the PAUT.

Figure 1 (a) shows input noisy PAUT testing ultrasonic signal. The signal is further subject to ALMS filter. The Fig. 1 (b) clearly shows the stage 1 of the filtered signal by eliminating random noise and Gaussian noise. The Fig. 1 (c) shows stage 2 of the filtered signal by eliminating noises. The Fig. 1 (d) shows the ROC curve of classification. The EWT transform here is applied to generally transform the time domain filtered signal into a frequency domain signal. The frequency domain signal is further subjected to 
feature extraction using 1 st order and 2 nd order feature extraction. Figure 1 graphical representation is for welding defective signal classification. The Fig. 2 shows the graphical representation for welding nondefective signal classification.

Table 1

\begin{tabular}{|llll|}
\hline S. No & Filters & SNR in dB & MSE \\
\hline 1 & Least Mean Square (LMS) type & 32 & 1.8748 \\
\hline 2 & Adaptive Least Mean Square type & 45 & 0.0938 \\
\hline
\end{tabular}

Table 2

\begin{tabular}{|llll|}
\hline S. No & Feature parameters & Welding non-defective & Welding defective \\
\hline 1 & Mean & 9 & 18 \\
\hline 2 & Standard Deviation & 8 & 16 \\
\hline 3 & Variance & 4 & 13 \\
\hline 4 & Co-variance & 3 & 15 \\
\hline 5 & Minima & 0 & 1 \\
\hline 6 & Maxima & 1 & 10 \\
\hline 7 & Entropy & 1 & 3 \\
\hline 8 & Homogeneity & 1 & 2 \\
\hline
\end{tabular}

The Table 1 shows the ALMS filter parameters such as Signal to Noise Ratio (SNR) and Mean Square Error (MSE). The Table 2 shows the various features that are being extracted using the 1 st order and 2 nd order feature extraction technique. The Table 3 shows the accuracy testing performed using Confusion Matrix (CM). As shown in Table 3, the DCNN efficiency and accuracy are better than BPNN. 
Table 3

Confusion Matrix (CM) accuracy testing

\begin{tabular}{|lllll|}
\hline S. No & Classification methods & Accuracy & Specificity & Sensitivity \\
\hline 1 & BPNN & 73 & 83 & 70 \\
2 & DCNN & 97 & 95 & 99 \\
\hline
\end{tabular}

\section{Conclusion}

In this research, phased array ultrasonic test (PAUT) signal enhancement and classification on carbon steel welding piece is implemented. The PAUT testing is proceeded for ultrasonic signal to detect welding defect. The PAUT NDT testing signal may contain various noises during testing of welding. The adaptive filter should be implemented to eliminate various noises. The Adaptive Least Means Square (ALMS) filter is applied to enhance the PAUT testing signal. The Empirical Wavelet Transform (EWT) technique is proposed in order to convert the time domain signal to its corresponding frequency domain signal. The feature extraction using 1st order and 2nd order techniques are applied on frequency domain signal. The Deep Convolution Neural Network (DCNN) is applied to classify welding defective signal and welding non-defective signal. The efficiency and accuracy of proposed methodology is better than existing methodology as shown in graph and tabulated numeral values.

\section{Declarations}

Ethics approval and consent to participate: Not applicable.

Funding: Not applicable.

Conflict of interests: The authors declare that they have no conflict of interests.

Informed Consent: Not applicable

Authors' contributions: All authors discussed the results and implications and commented on the manuscript at all stages. All authors read and approved the final manuscript for publication.

\section{References}

[1] M. Wahbeh, M. Foerder and R. Boundouki, "Phased array ultrasonic testing (PAUT) performed on electroslag weld specimen subject to cyclical tension load testing", DOI:10.3233/BRS-180124.

[2] J. Fortunato, Chirag Anand, Daniel F. O. Braga, R.M.Groves, P. M. G. P. Moreira and V. Infante, "Friction stir weld-bonding defect inspection using phased array ultrasonic testing", DOI: 10.1007/s00170-0170770-7. 
[3] A.Raude and T. Colombier, "Phased array inspection of aluminothermic rail welds The automated solution A fast convenient means of inspecting rail joints arrives".

[4] Xiaochuan Liu,Yu Wu,Zhenmao Chen,Cherdpong jom,Xiaobao Bai, Hongen Chen, Yunxi Jiang, Jinggang Qin, Changtao Cao, Aihua Xu, Qiaochu Wu and Houxiang Han, "Research on Nondestructive Examination of Bracket Welds of ITER In-Vessel Coils (IVC)", IEEE 2018.

[5]G. V. Shlyakhova, S. A. Barannikovaa, A.V. Bochkarevaa,Yu. V. Lia and L. B. Zuev, "Structure of a Carbon Steel-Stainless Steel Bimetal", DOI: 10.3103/S0967091218040101.

[6] Reza Mohammadkhani, Luca Zanotti Fragonara, Janardhan Padiyar M, Ivan Petrunin, João Raposo , Antonios Tsourdos and lain Gray, "Improving Depth Resolution of Ultrasonic Phased Array Imaging to Inspect Aerospace Composite Structures”, DOI:10.3390/s20020559.

[7] Uzzal Biswas, Anup Das, Saurov Debnath and Isabela Oishee,"ECG Signal Denoising by Using LeastMean-Square and Normalised-Least-Mean-Square Algorithm Based Adaptive Filter", IEEE 2014.

[8] Xing Kou, Cuixiang Pei, Shangzi Wu and Zhenmao Chen, "Phased-Array Laser Ultrasonic Testing with Sparse Total Focusing Imaging Method", IEEE 2019.

[9] Avinish Tiwari, Piyush Singh, Pankaj Biswas and Sachin D. Kore ,"Friction Stir Welding of Low-Carbon Steel",IEEE 2019.

[10] H. R. Ghazvinloo and A. Honarbakhsh Raouf, "Microstructure of The Weld Metal in Ck45 Carbon Steel", DOI 10.1007/s11003-019-00242-x.

[11] Dinu Thomas Thekkuden, Abdel-Hamid Ismail Mourad, "Investigation of feed-forward back propagation ANN using voltage signals for the early prediction of the welding defect", Springer 2019.

[12] Yunxia Xia, "Decomposition of Nonstationary Bridge Vibration Signals Using Empirical Wavelet Transform”, IEEE 2019.

[13] Zheyu Gao, Jing Lin, XiufengWang and Xiaoqiang Xu, "Bearing Fault Detection Based on Empirical Wavelet Transform and Correlated Kurtosis by Acoustic Emission”, IEEE 2017.

[14] Zhifen Zhang, Elijah Kannatey-Asibu Jr, Shanben Chen,Yiming Huang and Yanling Xu, "Online defect detection of Al alloy in arc welding based on feature extraction of arc spectroscopy signal", Springer 2014.

[15] T. Thaj Mary Delsy, N.M. Nandhitha, B. Sheela Rani, Feasibility of Recurrent Neural Network for the Binary classification of Non stationary signals, Microprocessors and Microsystems, Volume 82, 2021, 103955, ISSN 0141-9331, doi.org/10.1016/j.micpro.2021.103955. 
[16] T. Thaj Mary Delsy, N. M. Nandhitha \& B. Sheela Rani. Feasibility of spectral domain techniques for the classification of non-stationary signals. J Ambient Intell Human Comput 12, 6347-6354 (2021). https://doi.org/10.1007/s12652-020-02220-7

\section{Figures}

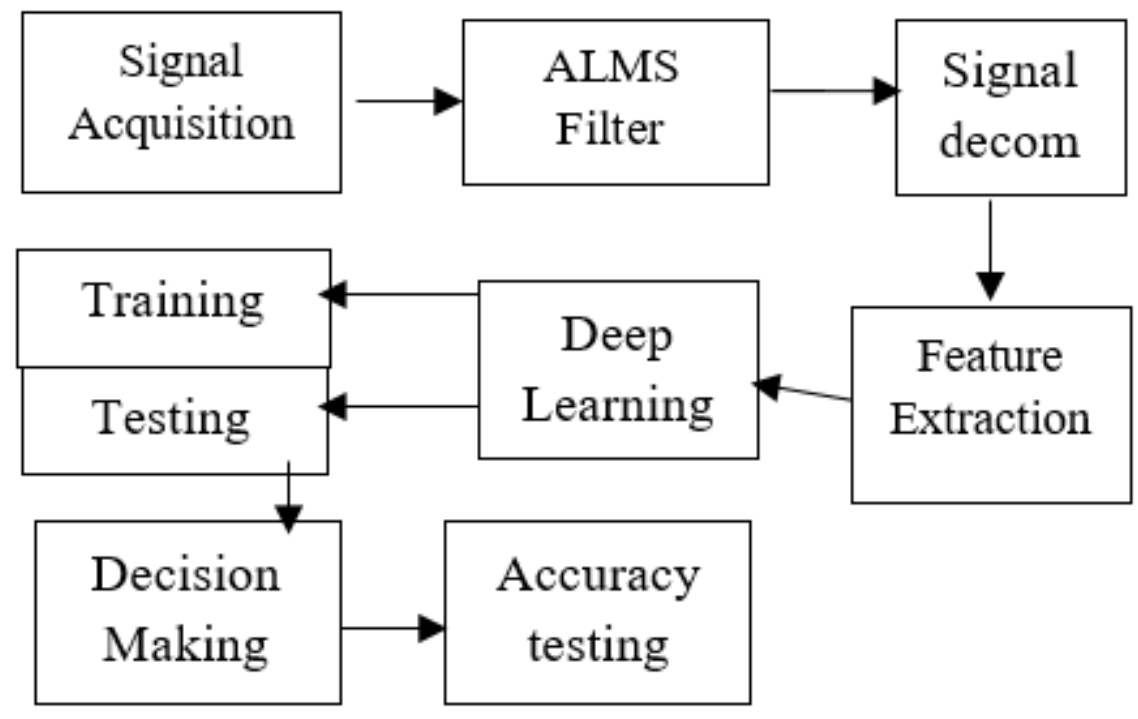

Figure 1

Architecture diagram of proposed methodology 


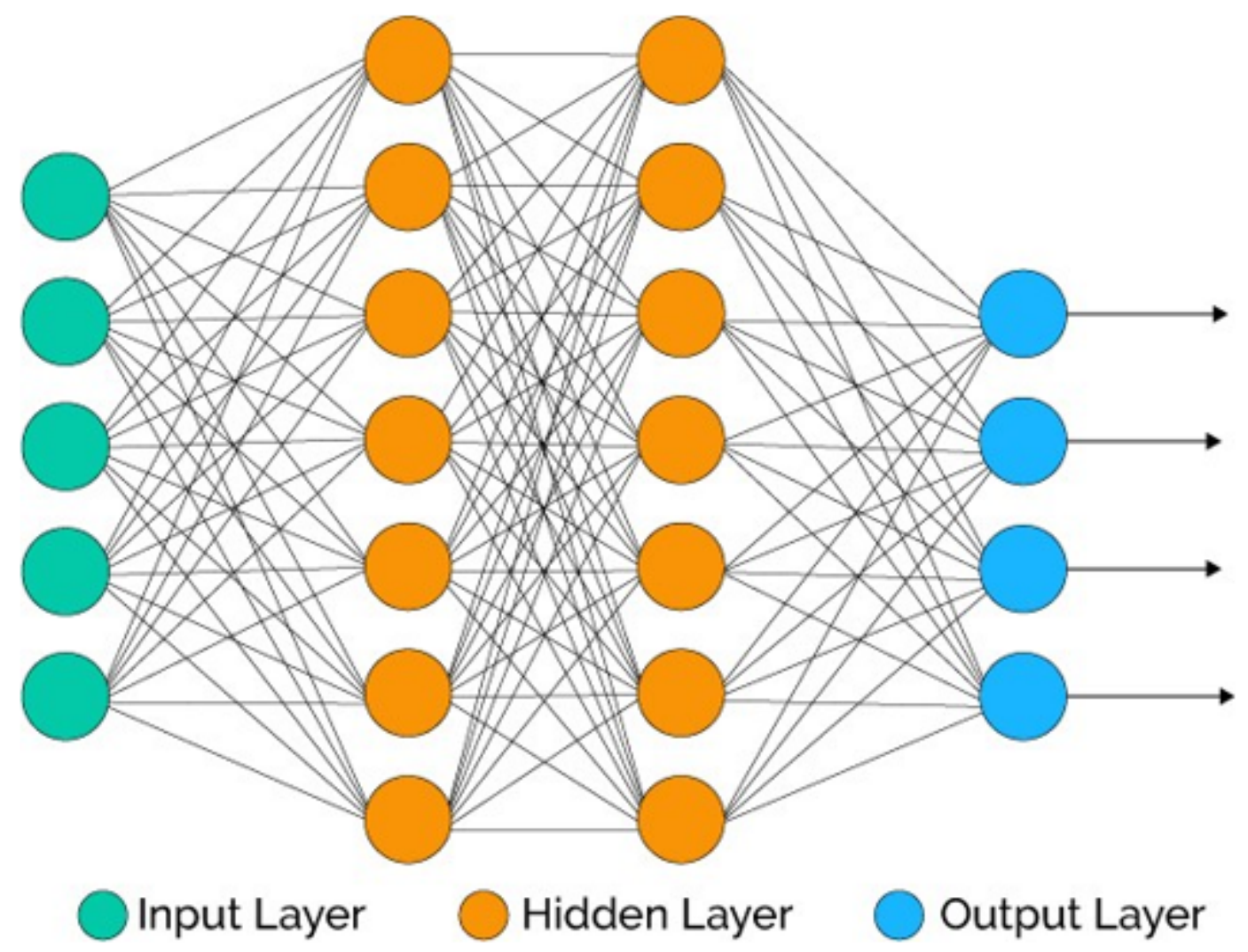

Figure 2

Deep Convolutional Neural Network (DCNN) Architecture 

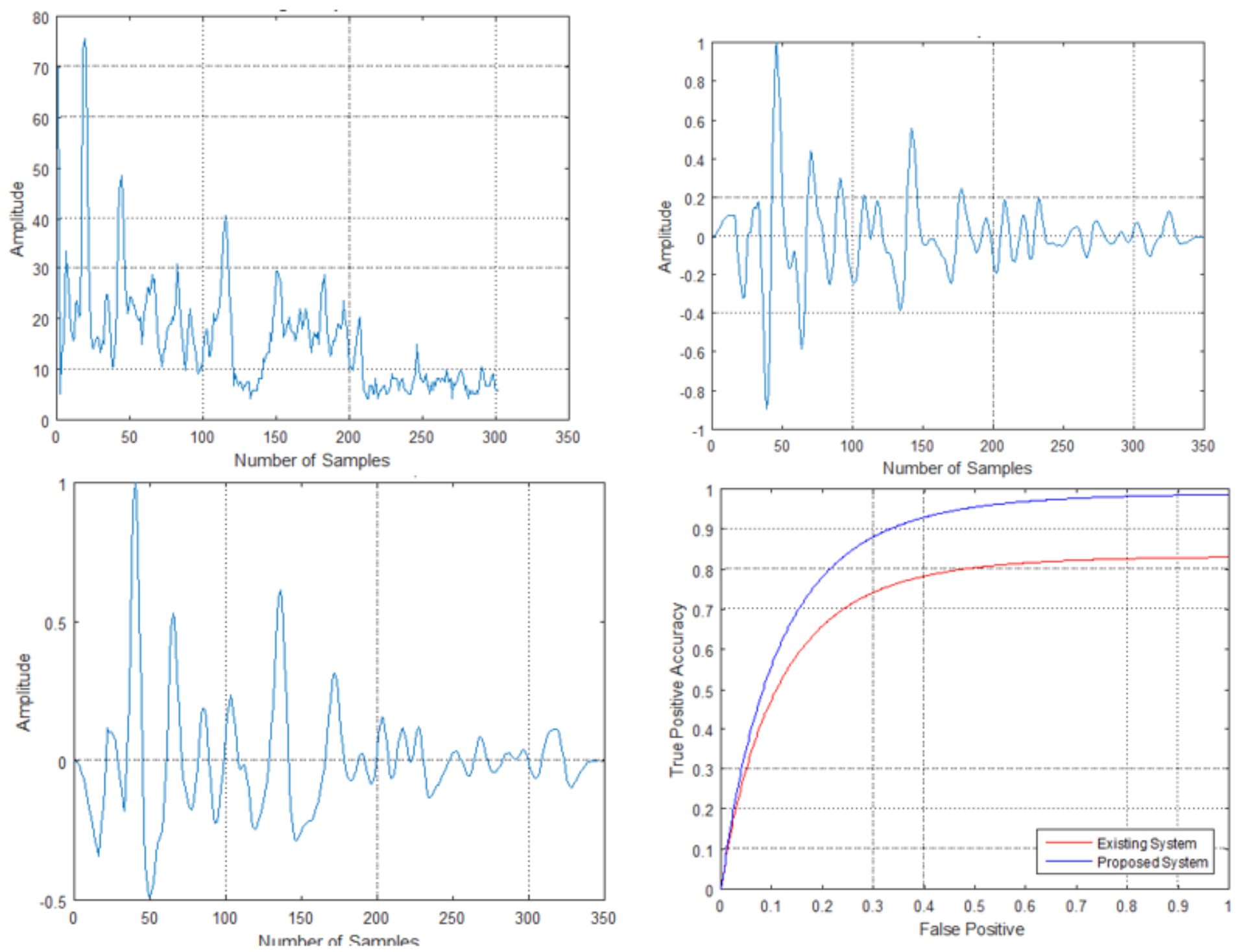

Figure 3

(a) Input noisy PAUT testing ultrasonic signal (Welding defective signal), (b) Filtered signal Stage 1 using ALMS, (c) Filtered signal Stage 2 using ALMS, (d) ROC curve 

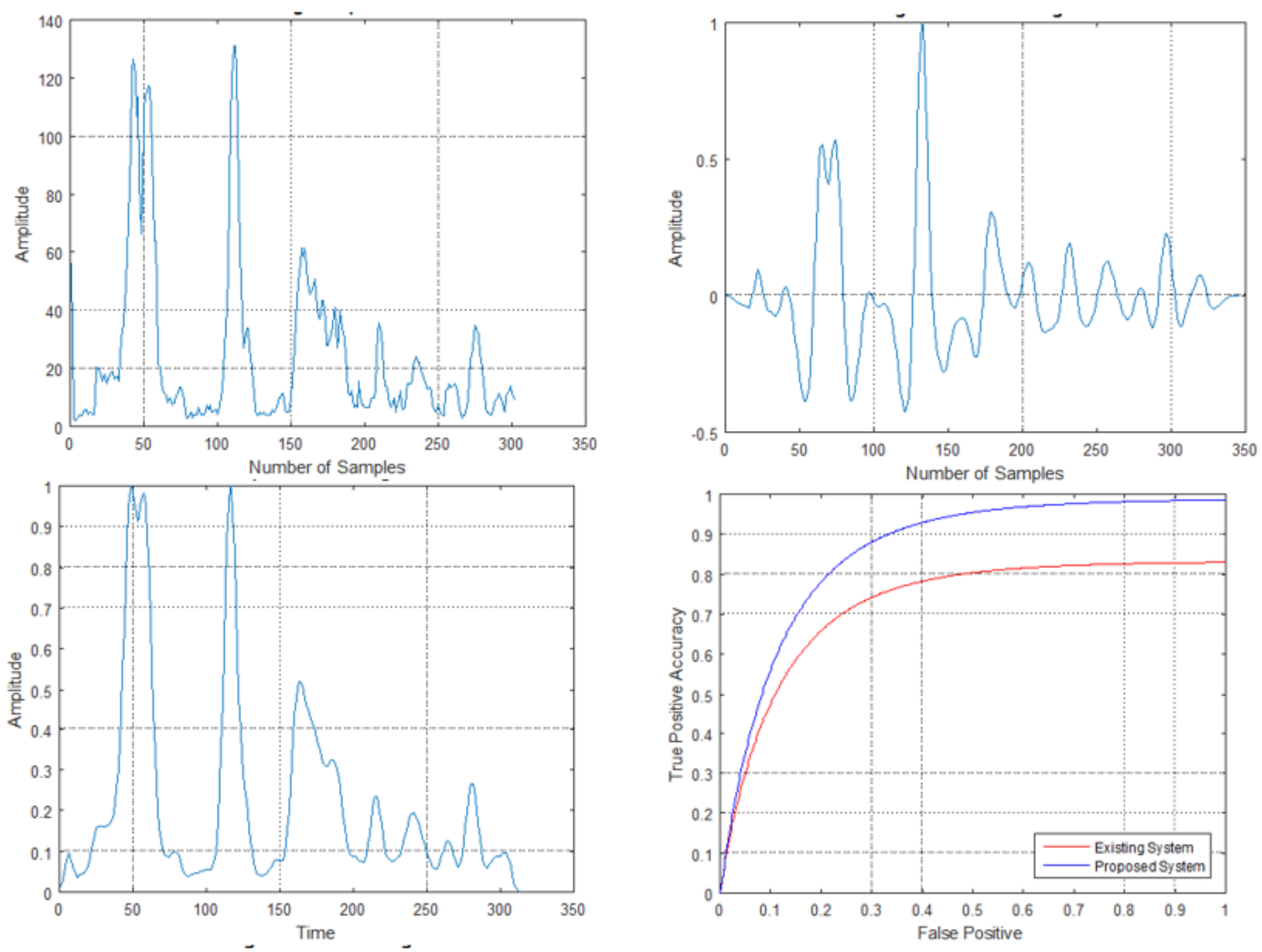

Figure 4

(a) Input noisy PAUT testing ultrasonic signal (Welding normal signal), (b) Filtered signal Stage 1 using ALMS, (c) Filtered signal Stage 2 using ALMS, (d) ROC curve 